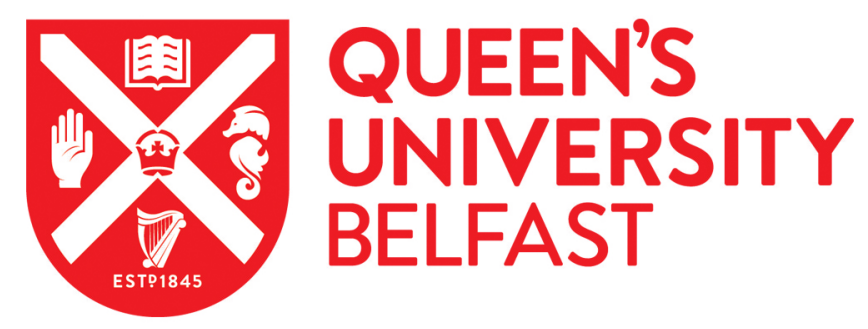

\title{
Three-dimensional Simulations of Detonation Propagation in Circular Tubes: Effects of Jet Initiation and Wall Reflection
}

Chen, W., Liang, J., Cai, X., \& Mahmoudi, Y. (2020). Three-dimensional Simulations of Detonation Propagation in Circular Tubes: Effects of Jet Initiation and Wall Reflection. Physics of Fluids, 32(4), [046104 ].

https://doi.org/10.1063/1.5143105

Published in:

Physics of Fluids

Document Version:

Peer reviewed version

Queen's University Belfast - Research Portal:

Link to publication record in Queen's University Belfast Research Portal

Publisher rights

Copyright 2020 AIP. This work is made available online in accordance with the publisher's policies. Please refer to any applicable terms of use of the publisher.

\section{General rights}

Copyright for the publications made accessible via the Queen's University Belfast Research Portal is retained by the author(s) and / or other copyright owners and it is a condition of accessing these publications that users recognise and abide by the legal requirements associated with these rights.

Take down policy

The Research Portal is Queen's institutional repository that provides access to Queen's research output. Every effort has been made to ensure that content in the Research Portal does not infringe any person's rights, or applicable UK laws. If you discover content in the Research Portal that you believe breaches copyright or violates any law, please contact openaccess@qub.ac.uk. 


\section{Three-dimensional Simulations of Detonation Propagation in Circular Tubes: Effects of Jet Initiation and Wall Reflection}

Weiqiang Chen (陈伟强) $)^{1,2}$, Jianhan Liang (梁剑寒 $)^{2}$, Xiaodong Cai (蔡晓东) ${ }^{2 *}$, Yasser Mahmoudi $^{3}$

${ }^{1}$ Science and Technology on Scramjet Laboratory, China Aerodynamics Research and Development Center, Mianyang, Sichuan 621000, China

${ }^{2}$ Science and Technology on Scramjet Laboratory, College of Aerospace Science and Engineering, National University of Defense Technology, Changsha 410073, China

${ }^{3}$ School of Mechanical and Aerospace Engineering, Queen's University Belfast Belfast BT9 5AH, United Kingdom

*Corresponding author: xdcaiaero@sina.com 
Abstract: In the present work, using a high-resolution three-dimensional numerical analysis the initiation and propagation mechanism of a detonation wave is studied in a circular tube with a hot jet initiation. The reactive Euler equations with a one-step two-species chemistry model are solved based on the structured adaptive mesh refinement technique. Influences of both a single hot jet and impinging double hot jets on the formation of the detonation wave are studied. For each case, the objective is to investigate the role of the tube wall on the initiation and propagation of the detonation wave. The result for both cases shows that the hot jet-induced bow shock forms a complex reflection structure in the circular tube. The reflection effect of the circular wall strengthens the shock and facilitates the formation of the Mach stem, which leads to the formation of the detonation wave. However, when the hot jet condition and the total area of jet hole remain the same, for the case of initiation using double hot jets, the reflection strength of the bow shocks weakens when the diameters of the hot jets become smaller. When using a single hot jet, the initiated detonation is overdriven and propagates in the twoheaded mode. In this initiation mode, by increasing the inflow Mach number, a four-headed mode detonation front is formed. While in the case of impinging double hot jets, a four-headed mode detonation front is initiated directly.

Keywords: Three-dimensional detonation; Circular tube; Initiation and propagation; Hot jet initiation; Wall reflection

\section{Introduction}

A detonation is a coupled shock and combustion wave propagating through a reactive mixture at supersonic speed. ${ }^{1}$ In addition to the main Mach stem and incident wave in the flow direction, a detonation consists of transverse waves that move along the circumferential and radial directions. The desire for high thermodynamic efficiency has motivated research on detonations for advanced 
propulsion system applications..$^{2-9}$ Therefore, deployment detonation combustion in supersonic flow in a scramjet may be an alternative choice for advanced propulsion systems. Most flight systems have an axisymmetric cylindrical shape, thus the adoption of a circular tube for detonation-based engines is a more practical choice. Many researchers have investigated the properties of detonation in circular tubes in quiescent or low-speed mixtures for pulsed detonation engines ${ }^{10}$ and continual rotating detonation engines ${ }^{11}$ to provide useful fundamental and practical data. Cho et al. ${ }^{12}$ investigated different modes of detonation wave propagation in a circular tube in quiescent mixtures with the pre-exponential factor as a controlling parameter. However, the details of the three-dimensional initiation and propagation mechanism in a circular tube in supersonic flow remain unclear.

Detonation initiation is one of the most important issues in detonation application. ${ }^{13}$ In supersonic flow and in comparison to other initiation mechanisms, a hot jet can rapidly initiate a detonation wave with a small amount of energy. ${ }^{14}$ In the past numerous studies have focused on the detonation initiation using a hot jet in quiescent or low-speed combustible mixtures (e.g. Ref ${ }^{15-20}$ ), whereas few studies have focused on supersonic combustible flows (e.g. $\operatorname{Ref}^{21-23}$ ). Cai et al. ${ }^{24}$ found that the sidewalls in a three-dimensional rectangular channel can help realize detonation initiation in supersonic combustible mixtures. However, few studies have focused on detonation initiation in a circular tube, which is more general in practical applications. The initiation mechanism needs to be further investigated.

Controlling the propagation mechanism of the detonation wave $\mathrm{e}^{25-27}$ is another key issue for having a reliable and efficient detonation-based propulsion system. Several propagation modes have been observed in quiescent mixtures in the detonation of a circular tube, such as the spinning, twoheaded, and multi-headed modes. ${ }^{1,28}$ Single-spinning detonation has only one transverse wave along the circumference, whereas two-headed detonation has two transverse waves along the circumferential direction and one transverse wave in the radial direction. ${ }^{29}$ Campbell and Woodhead ${ }^{30}$ first 
experimentally observed spinning detonation in a circular tube near the detonation limit. Several researchers, such as Huang, ${ }^{31}$ found that the shock structure of the spinning mode contains a complex Mach interaction. Oran, Gamezo and their collaborators ${ }^{32,33}$ have performed studies over three decades to determine the detailed detonation structure and its dynamics. Williams et al., ${ }^{34}$ Tsuboi et al., ${ }^{35}$ and Wang et al. ${ }^{36,37}$ conducted three-dimensional simulations in rectangular tubes to reveal its two modes, namely rectangular mode and diagonal mode. In a rectangular channel, Deiterding et al. ${ }^{38}$ efficiently simulated three-dimensional detonations using the adaptive mesh refinement code, AMROC (Adaptive Mesh Refinement in Object-oriented $\mathrm{C}++) .{ }^{39-42}$ Tsuboi et al., ${ }^{43}$ using three-dimensional simulations and a detailed chemical reaction model, analysed spinning detonations in both circular and square tubes and studied the characteristics of the spinning modes. They ${ }^{43}$ used a typical ZND detonation structure as initial condition and neglect the initiation process. Tsuboi et al. ${ }^{44}$ also investigated the energy release effect and found the effects of energy release are large on the strength of the transverse detonation. Sugiyama et $a l .{ }^{45}$ investigated the effect of activation energy on the spinning detonation. Virot et al. ${ }^{46}$ also studied the spinning detonation and obtained reasonable agreement with experimental results. These studies on the spinning detonation provide a deeper understanding of the detonation dynamics and propagation mode. Further investigations on the propagation mode in circular tubes must be performed for practical detonation applications in supersonic flows.

Therefore, the main objective of this work is to provide a fundamental understanding of the detailed initiation process and the dynamics of the initiated detonation front in a circular tube for supersonic combustible mixtures to facilitate detonation applications in a supersonic combustion chamber. The remainder of this paper is organized as follows: The numerical modeling is presented in Section 2. The adaptive mesh refinement is verified in Section 3. The results of the detonation simulation are provided in Section 4, in which the initiation mechanism and propagation mode are 
studied by comparing single and double jets. Finally, the conclusions of this paper are presented in Section 5.

\section{Numerical modeling}

\subsection{Governing equations and numerical methods}

Fig. 1 shows a diagram of computation model. The three-dimensional reactive compressible flow simulations utilize the Euler equations as governing equations. An irreversible one-step, two-species chemistry model is considered to avoid the complexity of viscous flow physics and detailed kinetics. The governing equations are expressed as follows:

$$
\frac{\partial \boldsymbol{U}}{\partial t}+\frac{\partial \boldsymbol{F}}{\partial x}+\frac{\partial \boldsymbol{G}}{\partial y}+\frac{\partial \boldsymbol{H}}{\partial z}=\boldsymbol{S}_{\text {chem }}
$$

where the conservative variable vector $\boldsymbol{U}$, the convective flux vectors $\boldsymbol{F}, \boldsymbol{G}$ and $\boldsymbol{H}$, the source term vector $\boldsymbol{S}_{\text {chem }}$, are, respectively, given as:

$$
\begin{gathered}
\boldsymbol{U}=\left(\rho, \rho u, \rho v, \rho w, \rho e_{t}, \rho Y_{1}\right)^{T} \\
\boldsymbol{F}=\left(\begin{array}{c}
\rho u \\
\rho u^{2}+p \\
\rho u v \\
\rho u w \\
\left(\rho e_{t}+p\right) u \\
\rho u Y_{1}
\end{array}\right), \boldsymbol{G}=\left(\begin{array}{c}
\rho v \\
\rho u v \\
\rho v^{2}+p \\
\rho v w \\
\left(\rho e_{t}+p\right) v \\
\rho v Y_{1} \\
\boldsymbol{S}_{\text {chem }}
\end{array}\right), \boldsymbol{H}=\left(\begin{array}{c}
\rho w \\
\rho u w \\
\rho v w \\
\rho w^{2}+p \\
\left(\rho e_{t}+p\right) w \\
\rho w Y_{1}
\end{array}\right)
\end{gathered}
$$

Here $\rho$ is the total density, $u, v$ and $w$ are the velocity in $x$-direction, $y$-direction and $z$-direction, $Y_{1}$ is the mass fraction of the reactant, $p$ is the pressure, $e_{t}$ is the total energy per unit mass, $\dot{\omega}_{1}$ is the mass production rate of the reactant.

Fig. 1. Schematic of the computational domain.

The total energy $e_{t}$ is defined as

$$
e_{t}=\frac{p}{(\gamma-1) \rho}+\frac{1}{2}\left(u^{2}+v^{2}+w^{2}\right)+Y_{1} q
$$


where $q$ is the heat release per unit mass.

Here the reaction model is selected and fitted to the physical parameters of a stoichiometric $\mathrm{H}_{2} / \mathrm{O}_{2}$ detonation initially at $\mathrm{T}=300 \mathrm{~K}$ and $\mathrm{P}=6.67 \mathrm{kPa}$, which defines a marginally stable detonation. The Zel'dovich-von Neumann-Döring (ZND) solution calculated with detailed chemistry is used to make an approximate chemistry and transport model. The Arrhenius rate, activation energy, pre-exponential, heat release and specific heat ratio are calculated by matching the Chapman-Jouguet (CJ) speed and the von Neumann pressure at the front of the ZND detonation.

For the two-species, calorically perfect model we have

$$
\gamma=\gamma_{1}=\gamma_{2}, p=\rho R T, R=R_{1}=R_{2}
$$

The mass fraction production rates are given as follows:

$$
\dot{\omega}_{1}=-\dot{\omega}_{2}=\rho Y_{1} A \exp \left(\frac{E_{a}}{R T}\right)
$$

Currently, it is very expensive to conduct high-resolution multi-dimensional detonation simulations solving the NS equations with detailed chemistry. If solving the NS equations, a turbulent boundary layer would develop along the circular tube walls and its thickness will strongly depend on the length of the inflow section of the circular tube. Especially, when the jet induced bow shock interacts with the boundary layer, shock wave-boundary layer interactions in combustible mixtures ${ }^{47}$ will happen. Thus, the initiation process is rather complicate and can only accurately be described by simulating the entire combustor chamber. In order to eliminate this complexity and to be able to focus the present study just on the jet ignition process and immediately subsequent detonation propagation in the circular tube. we have opted to use the Euler equations as governing equations and ignore the influence of boundary layers in our investigations.

One of the failures of the one-step model is its inability to capture the chain-branching character of the reactions and the corresponding cross-over effect. ${ }^{48}$ This can provide a change in the shock 
sensitivity of the reaction rates if the shock strength falls below a critical value. Nonetheless, there are additional issues that make the simulation of gaseous detonations challenging. These include the threedimensional nature of the coherent structures and the challenge of carrying out high-order simulations needed for turbulence modelling and simultaneously capturing shock waves, and finally the storage requirements for detailed chemical reaction mechanisms. ${ }^{49}$ Thus, most detonation simulations have been carried out basing on the reactive Euler equations with a simplified chemical kinetic model ${ }^{50,51}$ Even with simple reaction model, the results are in remarkable qualitative agreement with the experimental observations. ${ }^{52}$ Therefore, for many detonation simulations, the Arrhenius law that relates chemical reaction rates to temperature variation is widely used as the simplest mode. ${ }^{47,53}$

The details of the governing equations and the thermodynamic parameters of the one-step, twospecies chemistry model can also be found in studies by Ziegler, ${ }^{54}$ and the reaction model is discussed in-depth in studies by Bane. ${ }^{55}$ Table 1 presents the thermodynamic parameters of the chemistry model used in the simulations. The adoption of this simple model and the adaptive mesh refinement method provide advantages in terms of the computational efficiency for the heavy three-dimensional calculations.

Table 1 Thermodynamic parameters of the chemistry model

\begin{tabular}{lll}
\hline Parameters & Values & Unit \\
\hline Temperature $\left(\mathrm{T}_{\infty}\right)$ & 300 & $\mathrm{~K}$ \\
Pressure $\left(\mathrm{P}_{\infty}\right)$ & 6.670 & $\mathrm{kPa}$ \\
Density: $\left(\rho_{\infty}\right)$ & 0.077552 & $\mathrm{~kg} / \mathrm{m}^{3}$ \\
Specific heat ratio: $(r)$ & 1.29499 & - \\
Molecular weight of mixture: W & 0.029 & $\mathrm{~kg} / \mathrm{mol}$ \\
Heat release: q & 54000 & $\mathrm{~J} / \mathrm{mol}$ \\
Dimensionless heat release: q/RT & 21.65 & - \\
Activation energy: Ea & 30000 & $\mathrm{~J} / \mathrm{mol}$ \\
Dimensionless activation energy: Ea/RT $\infty$ & 12.028 & - \\
Zel'dovich number: Zn & 1.27 & - \\
Pre-exponential factor: A & $6 \times 10^{5}$ & $\mathrm{~s}^{-1}$ \\
Velocity of C-J state: $\mathrm{V}_{\mathrm{CJ}}$ & 1587.84 & $\mathrm{~ms}^{-1}$ \\
\hline
\end{tabular}


The second-order accurate MUSCL-TVD finite volume method is adopted for the convective flux discretization. The hydrodynamic solution of the hyperbolic system ${ }^{56}$ is separated into the flux calculation step and reconstruction step. Dimensional splitting is employed for the three-dimensional simulation. First-order Godunov splitting is adopted for the reactive source term and the standard onedimensional Roe-type Riemann solver is applied and then extended to three dimensions. A hybrid RoeHLL Riemann solver ${ }^{57}$ is utilized to circumvent the intrinsic problem of unphysical total densities and internal energies near vacuum due to the Roe linearization, ensuring positive mass fractions and preventing the disastrous carbuncle phenomenon. The Van Albada limiter is adopted with the MUSCL reconstruction to construct a second-order accurate TVD-type method along with the second-order accurate MUSCL-Hancock technique ${ }^{58}$ for temporal iteration. All simulations use the adjustment of dynamic time step with target Courant-Friedrichs-Lewy (CFL) number of 0.95 .

It is more efficient to use the high-order shock-capturing finite volume method on rectangular Cartesian grids. To construct the circular wall within the old frame, the ghost fluid method (GFM) ${ }^{59}$ is adopted, which uses finite volume cells that are inserted in the boundary condition as ghost cells. The boundary geometry is mapped onto the Cartesian mesh by employing a scalar level set function $\varphi$ that stores the signed distance to the boundary surface and allows for the efficient evaluation of the boundary outer normal at every mesh point. The level set function $\varphi$ is updated after every boundary synchronization step by calling the closest-point-transform algorithm, which was developed by Mauch. ${ }^{60}$ Because the method itself is not modified, a small amount of diffusion will be generated, resulting in an overall non-conservative numerical scheme. The simulations use the adaptive refinement method to refine the mesh near the circular wall to the highest refinement level in order to effectively alleviate the errors caused by this particular staircase boundary.

Initially, the circular tube consists of a stoichiometric mixture of $\mathrm{H}_{2} / \mathrm{O}_{2}$ reactant with a molar ratio 
$2: 1$ under the condition of $\mathrm{T}=300 \mathrm{~K}, \mathrm{P}=6.670 \mathrm{KPa}$ and $v=\mathrm{V}_{\mathrm{CJ}}=-1587.84 \mathrm{~m} / \mathrm{s}$. The supersonic flow direction in Fig. 1 is from right to left. The right boundary adopts a supersonic inflow condition. The incoming boundary condition was used with a stoichiometric mixture of $\mathrm{H}_{2} / \mathrm{O}_{2}$ detonation at $\mathrm{T}=300$ $\mathrm{K}$ and $\mathrm{P}=6.670 \mathrm{KPa}$ flowing at $\mathrm{V}_{\mathrm{CJ}}\left(\mathrm{V}_{\mathrm{CJ}}=1587.84 \mathrm{~m} / \mathrm{s}\right)$. The left boundary adopts an ideal supersonic outflow condition, where values are extrapolated with zero-gradient condition. Knowing that the focus of the present study is on the jet ignition process and the subsequent detonation propagation, we considered slip boundary conditions on the tube wall to allow the boundary layers produced in the inflow section to be neglected..$^{23}$ Thus, a reflecting boundary with slip wall conditions is used on the circular wall. The inflow of the hot jet is considered on the upper or lower jet channel. When the hot jets are shut down, the inflow condition switches to the reflecting condition immediately.

The inflow parameters of the hot jet in all cases are set to the values of the ZND state of a detonation in $\mathrm{H}_{2} / \mathrm{O}_{2}$ under an inflow pressure of $40.0 \mathrm{kPa}$ and a temperature of $300 \mathrm{~K}$ in all simulations. These conditions yield the following inflow parameters for the hot jets: $\mathrm{P}=596.334 \mathrm{kPa}, \mathrm{T}=2519.54$ $\mathrm{K}, \rho=0.825574 \mathrm{~kg} / \mathrm{m} 3$, total energy $=2.24021 \times 106 \mathrm{~J} / \mathrm{mol}$, mass fraction of $\mathrm{Y} 1$ (reactant) $=$ 0.00198911 , and mass fraction of $\mathrm{Y} 2$ (product) $=0.99801089$. The injection velocity is specified as the sonic speed to make it a choked hot jet with a velocity of $722.73 \mathrm{~m} / \mathrm{s}$.

\subsection{Computational setup}

As shown in Fig. 1, the computational domain studied is a circular tube with a diameter of $25 \mathrm{~mm}$ and a length of $150 \mathrm{~mm}$. For the initiation with a single hot jet on the upper wall, the hot jet has a diameter of $4 \mathrm{~mm}$ and for the case of impinging double hot jets, both hot jets have a diameter of 2.8 $\mathrm{mm}$, keeping the total area of the cross section the same as that of a single hot jet. The initial base grid for the computational domain is about 900,000 cells distributed in three directions as 300 (x-direction) $\times 60($ y-direction $) \times 50($ z-direction $)$. Three additional mesh refinements with the refinement factors 
are 2, 2, and 2 are deployed. The corresponding uniform mesh number to the present adaptive mesh resolution is about 450 million cells. Verification of the grid resolution is presented in the next section. The 3D computations are conducted on Tianhe-II HPC at National Supercomputer Center in Guangzhou with 480 Intel E5-2692 $2.20 \mathrm{GHz}$ (Ivy Bridge) processors. A typical clock time for the simulations presented in this work is about five days.

\section{Verification of the adaptive mesh refinement}

Using the present method, Ralf Deiterding has conducted the three-dimensional cellular structure simulations and verified the reliability of the parallel AMR algorithm. ${ }^{39}$ His results are in perfect agreement with other numerical results and experiments. Cai has conducted a series of numerical simulations on detonation initiation and propagation in supersonic combustible mixtures using the open-source program AMROC. ${ }^{24,42,61}$ These series studies fully verified the effectiveness of the program.

To validate the current simulation, the numerical result of the reflecting structure is compared with our experimental observation. The experiment was conducted on the supersonic detonation research platform in our group. ${ }^{14}$ The exit diameter of the hot jet in the experiment is the same with the present simulation of single hot jet initiation. A shadowgraph system is adopted to observe the processes of detonation initiation.

Fig. 2 shows the comparison between the experimental result and the numerical simulation. As shown in Fig. 2, it is found that the overall structure of the numerical result is the same with the experiment observation, especially the bow shock, shear vortex structure and transverse wave. On the other hand, there are also some differences which is clearly to distinguish from the results. In Fig.2(a), the bow shock interacts with boundary layer, forming an oblique shock, while in Fig.2(b), a Mach stem 
is formed in the simulation.

From the comparisons between the numerical density schlieren image and the experimental shadowgraph, it is indicated that the numerical results agree basically with the experimental observations. It should be noted that the inviscid Euler equations are not able to simulate the complex shock boundary layer interactions in the experimental shadowgraph, which is waiting for the calculation of the N-S equations.

Fig. 2. Comparison between experimental result and numerical simulation. (a) experimental shadowgraph, (b) numerical schlieren of vertical center section calculated from xoy plane.

Previous research (e.g. $\operatorname{Ref}^{35}$ ) found that $10 \mathrm{Pts} / L_{1 / 2}$ (grid number within half reaction zone length) can reproduce the unstable behavior in a one-dimensional ZND test, and $20-40 \mathrm{Pts} / L_{1 / 2}$ are necessary in two-dimensional ZND detonation simulations. Williams, Bauwens and Oran ${ }^{34}$ studied the 3D structure of the ZND detonation with a resolution of $16 \mathrm{Pts} / L_{1 / 2}$. Deiterding ${ }^{38}$ considered the case with a resolution of $30 \mathrm{Pts} / L_{1 / 2}$ using the adaptive mesh refinement method. In our previous work, ${ }^{24}$ we use an effective resolution of $30.85 \mathrm{Pts} / L_{1 / 2}$ to investigate the three-dimensional detonation initiation in rectangular channel. In the present work, a much higher grid resolution is considered in order to properly resolve the initiation and propagation mechanism of the detonation with hot jet initiation. The base grid resolution is considered to be $0.5 \mathrm{~mm} \times 0.5 \mathrm{~mm} \times 0.5 \mathrm{~mm}$. Three additional refinement levels are adopted with the highest grid resolution to be $0.0625 \mathrm{~mm} \times 0.0625 \mathrm{~mm} \times 0.0625 \mathrm{~mm}$. Under the present flow condition, the half reaction length of the one-dimensional ZND model is $L_{1 / 2}=3.5369 \mathrm{~mm}$, which was calculated by Cantera. ${ }^{62}$ Thus, the highest mesh resolution here is about $57 \mathrm{Pts} / L_{1 / 2}$. The grid resolution is higher than those considered in previous investigations, indicating that the grid resolution used in the present work can reasonably achieve reliable results. 
To validate the convergence of current mesh resolution, the higher resolution of numerical simulation is conducted. Considering our computational resources, we conducted the simulation in two-dimensional rectangular channel for higher grid resolution of $114 \mathrm{Pts} / L_{1 / 2}$. The height of the channel is equal to the diameter of the circular tube. The inflow parameters of the hot jet, the initial and boundary conditions are the same with the three-dimensional simulation of single hot jet initiation.

Fig. 3 shows the comparison of initiation feature between the resolution of $57 \mathrm{Pts} / L_{1 / 2}$ and 114 Pts/L1/2. As shown in Fig. 3, it is found that the overall structure of the numerical result is the same with higher mesh resolution, especially the bow shock, transverse wave and the Mach stem induced detonation. From the comparisons between the two different mesh resolutions, it is indicated that the wave features are resolved well using resolution of $57 \mathrm{Pts} / L_{1 / 2}$.

Fig. 3. Comparison of the initiation feature between (a) $57 \mathrm{Pts} / \mathrm{L}_{1 / 2}$ and (b) $114 \mathrm{Pts} / \mathrm{L}_{1 / 2}, 1$-bow shock, 2-transverse wave, 3-Mach stem induced detonation.

In conclusion, the resolution utilized in this work is significantly higher than those deployed in the literature for 3D simulation of detonation wave, which produce real feature of detonation wave.

The numerically reproduced density schlieren images and the corresponding adaptive level distributions in the circular tube simulation at two different times are shown in Fig. 4, illustrating the capability of the AMR technique used in the present work to capture properly the fine details of the 3D detonation structure. The mesh adaptation is four-level refinement, represented by four different colors. The red color shows the highest fourth refinement level, and the blue color shows the base level. An optimal combination of mesh adaption flag parameters should be established for the problem at hand. Here, we used the threshold values of $\varepsilon_{\rho}=2 \times 10^{-1} \mathrm{~kg} \cdot \mathrm{m}^{-3}, \varepsilon_{p}=2 \times 10^{5} \mathrm{~Pa}$, and $\varepsilon_{\text {product }}=5 \times 10^{-2}$ for density, pressure and product scaled gradient, respectively, for the adaptive mesh refinement of the 
reaction front. In Fig. 4(a), Mach reflection is clearly formed when the jet-induced bow shock reflects on the lower wall of the circular tube. The shock structures, which consist of the incident bow shock, Mach stem and transverse wave, are resolved with the highest refinement level. In Fig. 4(b), the Mach stem structure propagates upstream and gets higher. The Mach stem structure is captured precisely by the dynamic adaptive mesh with the highest refinement level, efficiently realizing the AMR deployed in the simulation of the circular tube. Moreover, all of the circular walls are refined to the highest mesh refinement level at different times and thus effectively alleviate the errors caused by the ghost fluid method (GFM).

Fig. 4. Density schlieren images (left) and corresponding adaptive level distributions (right). (a) $t=$

$$
105 \mu \mathrm{s}, \text { (b) } t=180 \mu \mathrm{s} \text {. }
$$

\section{Results and discussion}

\subsection{Single hot jet initiation}

\subsubsection{Shock reflection on the circular tube}

The density isosurface of $0.09 \mathrm{~kg} / \mathrm{m}^{3}$ in Fig. 5 illustrates the shock reflection structure on the circular tube. Different colors of dashed lines represent the triple lines between different shocks. When the hot jet injects into the supersonic flow in the circular tube, a bow shock will form. The bow shock interacts with the left or right side wall and then reflects from the lower wall of the tube. The reflected shock formed on the sidewall is highlighted in Fig. 5. However, only shock-induced combustion occurs because the reflected shock is still not sufficiently strong. Moreover, the jet is sufficiently strong to induce the bow shock to reflect from the lower wall. A Mach stem surface that is perpendicular to the tube wall is formed. The Mach stem is stronger than both the bow shock and shock reflected from the sidewall. From the lower wall to the sidewall, the height and strength of the Mach stem decreases. The 
Mach stem then induces the detonation from the lower zone, where the shock is strongest to burn the mixture quickly. In the initiation process, the lower wall of the circular tube plays an important role in forming the Mach stem. The initiation process is slightly different from those in reference ${ }^{24}$, where the effect of reflection from the sidewall in the rectangular tube helps to generate Mach stem-induced detonation (MSID).

Fig. 5. Reflection structure of the jet-induced bow shock in the circular tube, $t=133 \mu \mathrm{s}$.

\subsubsection{Development of the detonation}

The instantaneous structure of the hot jet-initiated detonation in the circular tube is shown in Fig.

6. When a hot jet injects into the main flow, the jet-induced bow shock is not sufficiently strong to initiate the mixture directly. The bow shock reflects off the circular tube wall and forms a complex reflection structure, inducing a detonation near the lower wall of the circular tube. As shown in Fig. 6(a), the hot jet-induced bow shock forms three main different zones: the hot jet combustion zone, the Mach stem-induced combustion zone and the unreacted zone. In Fig. 6(b), the yellow dashed line denotes the hot jet combustion front. The Mach stem-induced combustion front consists of the detonation front and the shock-induced combustion front, which are denoted by the red and pink dashed line, respectively. When injecting into the main flow, the hot jet-induced bow shock is sufficiently strong to form a Mach reflection on the circular wall. In comparison to the reflection from the left and right sides, the reflection is stronger on the lower side. Thus, MSID occurs on the lower side, where the reactants are burned out behind the Mach stem. At the outlet, the reactant has entirely converted into product. However, on the right and left sides of the tube, the reflected shock is not sufficiently strong to induce a detonation wave, hence leads to the appearance of unreacted gases at the tube outlet. 
Fig. 6. Instantaneous structure of hot jet initiation at $t=133 \mu \mathrm{s}$, (a) three slices on the contour of the product and density isolines, (b) schematic of the shock front and combustion front structure.

Fig. 7 shows the instantaneous density and the product distributions of flow fields at vertical and horizontal central sections. Fig. 7(a) clearly shows the Mach reflection structure formed by the jetinduced bow shock surface on the lower wall. Fig. 7(b) shows the Mach reflection structures formed by the bow shock surface on sidewalls of the circular tube. It can be seen from Fig. 7(e) that the peak density along line 'AA' is $0.53 \mathrm{~kg} / \mathrm{m}^{3}$, which is much larger than the peak density of along line 'DD' $\left(0.31 \mathrm{~kg} / \mathrm{m}^{3}\right)$. This shows that compared with the position 'DD', along the position 'AA', the reactant is compressed more strongly by the bow shock. Both the position along 'BB' and the position along'EE' are Mach reflection regions. The density peak value of $0.45 \mathrm{~kg} / \mathrm{m}^{3}$ along 'BB' is also greater than the density peak value of $0.41 \mathrm{~kg} / \mathrm{m}^{3}$ along 'EE', showing that the Mach reflection near the lower wall is stronger than that near the sidewalls. Fig. 7(c) shows that the bow shock surface forms the Mach steminduced detonation zone near the lower wall. While Fig. 7(d) shows that the Mach reflections on sidewalls form shock induced combustion. As can be seen from Fig. 7(f), in the unreacted channel of the lower wall surface and the sidewall surface, the highest values of product along the lines 'aa' and 'dd' are only 0.26 and 0.12 , respectively. The peak value along 'bb' is 0.96 . As can be seen from the bb line in Fig. 7(f), after a distance of about $3.5 \mathrm{~mm}$, half of the reactant become product, which is almost the same as the theoretically calculated half reaction length of the one-dimensional ZND model $\left(L_{1 / 2}=\right.$ $3.5369 \mathrm{~mm}$ ). This indicates that the Mach stem induced a detonation wave at the lower wall. In Fig. $7(f)$, the ee line reached its peak value at $x=0 \mathrm{~mm}$ (the exit of the tube), only 0.54 . The length of the half reaction length along line ee is $16.5 \mathrm{~mm}$, indicating that the bow shock reflected on the sidewalls only form shock-induced combustion. 
From the bottom side to the left or right side, the MSID combustion gradually changes to a shock-

Fig. 7. Instantaneous density and product distributions of the flow fields at $t=133 \mu \mathrm{s}$, (a) the contour of density at vertical center section (xoy plane), (b) the contour of density at horizontal center section (xoz plane), (c) the contour of product at vertical center section (xoy plane), (d) the contour of product at horizontal center section (xoz plane), (e) instantaneous density curves at five positions shown in (a) and (b), (f) instantaneous product curves at five positions shown in (c) and (d).

induced combustion. At the same time, the MSID occurs in the zone near the low wall. Moreover, the MSID is overdriven, facilitating the upstream propagation of the Mach stem. The Mach stem travels upward and the areas of MSID become larger. Finally, the entire detonation front forms under the coupled effect. The formation of the Mach stem is achieved by the reflection effect of the circular tube. The Mach stem continues to propagate upstream and successfully induces the entire detonation front.

\subsection{Double hot jet initiation with original jet velocity}

As shown in Fig. 8, the Mach-induced detonation front is acquired using two impinging hot jets in the upper and lower walls of the circular tube. The diameter of the circular tube, the inflow condition and the hot jet condition remain the same as those in the single hot jet case. The two impinging hot jets induce more complex shock fronts, which is divided into four main zones. The entire shock front is longitudinally symmetric and bilaterally symmetric. When two hot jets are injected into the main flow simultaneously, the jets will induce bow shocks (zone 1 in Fig. 8). The bow shocks collide with each other at the center and form a regular reflection, which is denoted by triple line 1 in Fig. 8 . The bow shocks also reflect on the sidewall and form a Mach reflection on the circular tube (zone 2 in Fig. 8).

The bow shocks also reflect together at the sides and form a Mach reflection (zone 3). The Mach 
reflection is strengthened by the circular tube and forms another Mach reflection in zone 4. The MSID occurs behind zone 4 and, hence, the shock in zone 4 has the highest pressure of the four zones.

The strength of the two hot jet-induced bow shocks weakens because the decrease in the hot jet diameter. Thus, the reflection of the bow shock at the center cannot initiate a detonation directly. Although the reflection of the bow shocks near the sidewall is weaker than that at the center, the additional reflection of the bow shock on the side wall strengthens the reflection and forms zone 4 . The mixture behind the Mach stem is initiated and forms the MSID. The Mach stems at the sidewall propagate upstream and successfully initiate the entire mixture. When using the impinging double hot jets, the sidewall of the circular tube plays an important role in detonation initiation. The sidewalls promote the jet-induced bow shock reflection and strengthen the Mach reflection.

Fig. 8. Shock front structure of the impinging double hot jet-induced bow shock reflection at $t=130 \mu$ s. MSID: Mach stem-induced detonation, (1):bow shock, (D:

Mach reflection on the circular tube, (3): Mach reflection by bow shocks interaction, (4): Mach reflection on the sidewall.

\subsection{Detonation propagation}

\subsubsection{Single hot jet-initiated detonation}

The initiated detonation front moves in the two-headed mode when using a single hot jet for initiation. The hot jet is shut down after successful imitation of the detonation at $t=200 \mu \mathrm{s}$ after the transverse wave collides on the top of the wall. The initiated overdriven detonation front propagates upstream. Detonation propagation in a circular tube differs from that in a rectangular tube. The triple point along the circumference will leave a continual cell on the tube wall. In the circular tubes, the number of main triple points along the circumference represents the propagation mode e.g., the two- 
headed mode corresponds to two main triple points along the circumference simultaneously and leaves an entire cellular structure on the tube wall $^{63}$. For the present condition, the initiated overdriven detonation propagates in the two-headed mode in the circular tube, as shown in Fig. 9. In each subfigure, the left figure is the front view, and the middle figure represents the rear-side view. The blue isosurface is the $0.09 \mathrm{~kg} / \mathrm{m}^{3}$ density isosurface, showing the detonation front and the triple lines. The white dashed lines also outline the triple lines. The green or lime yellow isosurface is the $245 \mathrm{kPa}$ pressure isosurface, showing the transverse wave zone back of the detonation front. The third figure is a schematic of the main triple point line of the two-headed mode.

In Fig. 9(a) a curving triple line is generated. The triple line becomes longer when it collides downward. Next, the triple line reflects on the wall, and two short triple lines form as the cross section decreases. The two sub-triple lines propagate face to face, as shown in Fig. 9(b). In Fig. 9(c) the two sub-triple lines continue to move and become longer, whereas the main triple line becomes shorter and reflects up from the lower wall. The intersection of the three triple lines causes a strong local explosion behind the detonation front. Next, the sub-triple lines collide and travel in an opposite direction, merging with the main triple line. The long main triple line appears again and propagates upward, as shown in Fig. 9(d). When the triple line collides up, it repeats the moving mode when it collides down, first becomes longer and then reflects on the wall, forming two short triple lines. Next, the two triple lines travel in the opposite direction and then join with the reflected main triple line again (Fig. 9(f)). The detonation front moving upstream collides periodically with the triple lines.

Fig. 9. Detonation propagation in the two-headed mode in a circular tube, (a) $t=220 \mu \mathrm{s}$, (b) $t=235 \mu \mathrm{s}$, (c) $t=245 \mu \mathrm{s}$, (d) $t=255 \mu \mathrm{s}$, (e) $t=265 \mu \mathrm{s}$, (f) $t=285 \mu \mathrm{s}$. The blue isosurface is the $0.09 \mathrm{~kg} / \mathrm{m}^{3}$ density isosurface, the green or lime yellow isosurface is the $245 \mathrm{kPa}$ pressure isosurface. 


\subsubsection{Effects of inflow Mach number}

To obtain a well-developed detonation front with the given tube length, the inflow velocity is increased to $1739.39 \mathrm{~m} / \mathrm{s}$ i.e., the overdriven factor is equal to 1.2 , if the initiated detonation holds at a fixed position in supersonic inflow. Moreover, the time when the hot jet shuts down is extended to $t$ $=300 \mu \mathrm{s}$, ensuring that the mixture will still be initiated under the higher inflow Mach number. The other settings remain the same as those of the single hot jet initiation case in section 4.1.

As shown in Fig. 10, as the inflow Mach number increased, the initiated detonation propagates with four-headed mode detonation. The detonation is a two-headed mode detonation only when the detonation is initiated. Although the inflow Mach number is increased, the initiated overdriven detonation can develop more fully in the present finite length of the circular tube. Thus, the initiated detonation finally transforms to the four-headed mode. In Fig. 10, the arrows denote the propagation direction of the transverse waves. The strong transverse waves ' $a$ ' and ' $b$ ' move face to face and will collide at the center of the tube. Waves 'c' and 'e' are part of the transverse wave 'a' moving along the wall with the movement of ' $a$ ', and ' $f$ ' and ' $d$ ' are included in ' $b$ '. Two sets of dual head transverse waves intersect with each other as the initiated detonation propagates. When using a single hot jet, for the given circular tube length and inflow parameter settings, the initiated detonation will develop into the four-headed mode in the finite length of the circular tube.

Fig. 10(c) shows the pressure distribution of the radial cross-section structure. Two transverse waves move face to face and form a keystone-like structure behind the detonation front. The transverse waves are short and have different strengths. In comparison to the results of the detonation structure in a rectangular channel (e.g. Ref ${ }^{24,35}$ ), the main characteristic of the detonation front in the circular tube is the curving of the triple line. The curved circular wall causes this behavior when the triple lines collide in the tube. The Mach stem face expands and compresses periodically by the curving wall when 
the triple lines collide up and down. Thus, the local explosion may be stronger than that in a rectangular channel. The location of the triple line along the circular wall indicates that the jet-initiated detonation front in the supersonic mixtures is more complex than the wave front achieved by the distribution of the ideal ZND solution.

Fig. 10. Four-headed mode detonation wave front structures obtained with a higher inflow Mach number at $t=680 \mu \mathrm{s}$ : (a) front structure, (b) rear-side structure, and (c) cross-sectional structure.

\subsubsection{Double hot jet-initiated detonation}

Fig. 11 shows a temporal sequence detonation front of the detonation initiated via impinging of two hot jets in the circular tube. The impinging double hot jet-initiated detonation propagates in the four-headed mode. Two transverse waves collide at the center of the tube, and a strong local explosion occurs at the lower wall of the tube. When colliding to the sidewall, a new transverse wave is generated and collides upward. Next, the new transverse wave continues to propagate up to the upper wall when the two older transverse waves collide from the sidewall. The transverse wave will cause a strong local explosion on the upper wall. A new transverse wave is generated from the upper wall, and the process repeats. The present cell mode is slightly different from that of the previous section. In the one jet initiation, there is two main transverse waves colliding each other. While in double hot-jet initiation, besides the two main transverse waves collide face to face or back to back, there is a new transverse wave collides vertically to two main transverse waves. This difference may be caused by the initiation manner of the hot jets. When using a single hot jet with higher inflow velocity, the initiated detonation is two head mode and develop into the four-headed mode. When using impinging of double hot jets, the four-headed mode detonation is initiated directly. The two transverse waves collide at the center of 
the tube, thus strong local explosion happens with the focus effect at the lower curved wall of the tube. As a result, a new transverse wave generates.

In the present inflow condition, the steady cell width of the $2 \mathrm{D}$ detonation is equal to the diameter of the circular tube. For the present setting, the four-headed mode is the steady mode of the detonation front. When using the impinging double hot jets, the reflection of the bow shocks will induce two-cell mode detonation fronts, whereas the single hot jet initiation requires a higher inflow velocity or may require a longer tube for full development. Impinging of the double hot jets can yield a four-headed mode detonation more quickly in the finite length of the circular tube. Thus, the number of the hot jets and the injection method of the hot jets may play important roles in the formation of quasi-steady detonation. In the present condition, the impinging double hot jets achieve a quasi-steady detonation front more easily than a single hot jet.

Fig. 11. Four-headed mode detonation wave front structures and cross-sectional structures obtained by impinging double hot jets. (a) $t=410 \mu \mathrm{s}$, (b) $t=420 \mu \mathrm{s}$, (c) $t=430 \mu \mathrm{s}$, (d) $t=440 \mu \mathrm{s}$.

\section{Conclusions}

In this work, the three-dimensional reactive Euler equations with a one-step, two-species chemistry model were solved using the adaptive mesh refinement method in order to investigate the initiation and propagation of a detonation in a circular tube in supersonic combustible flows. The results showed that when using a single hot jet for initiation, the hot jet-induced bow shock forms a complex reflection structure in the circular tube. The reflection effect of the circular wall strengthens the shock and facilitates the formation of the Mach stem. The Mach stem initiates the detonation. The detonation initiation is achieved by the classical adiabatic shock compression mechanism. In the case 
of initiation using a pair of impinging hot jets, the jet-induced bow shock divides into four main shock fronts. The single hot jet-initiated detonation is overdriven and propagates in a two-headed mode. Increasing the inflow Mach number for the single hot jet initiation case, results in the formation of a four-headed mode detonation front. When the total area of the cross section holds the same as that of a single hot jet, impinging double hot jets can also directly initiate a four-headed mode detonation front.

\section{Acknowledgments}

This work is supported by National Natural Science Foundation of China (grant numbers 11702323, 91541203) and the National Foundation of Defense Technology (grant number 3101032).

\section{References}

1. J. H. S. Lee, The detonation phenomenon (Cambridge University Press, New York, 2008).

2. K. Kailasanath, "Recent developments in the research on pulse detonation engines," AIAA J. 41, 145 (2003).

3. N. N. Smirnov, V. B. Betelin, V. F. Nikitin, Y. G. Phylippov, and J. Koo, "Detonation engine fed by acetylene-oxygen mixture," Acta Astronaut. 104, 134 (2014).

4. S. Miao, J. Zhou, S. Liu, and X. Cai, "Formation mechanisms and characteristics of transition patterns in oblique detonations," Acta Astronaut. 142, 121 (2018).

5. Y. Liu, W. Zhou, Y. Yang, Z. Liu, and J. Wang, "Numerical study on the instabilities in H2-air rotating detonation engines," Phys. Fluids 30, 046106 (2018).

6. Y. Fang, Y. Zhang, X. Deng, and H. Teng, "Structure of wedge-induced oblique detonation in acetylene-oxygen-argon mixtures," Phys. Fluids 31, 026108 (2019).

7. T. Wang, Y. Zhang, H. Teng, Z. Jiang, and H. D. Ng, "Numerical study of oblique detonation wave 
initiation in a stoichiometric hydrogen-air mixture," Phys. Fluids 27, 096101 (2015).

8. P. Yang, H. D. Ng, H. Teng, and Z. Jiang, "Initiation structure of oblique detonation waves behind conical shocks," Phys. Fluids 29, 086104 (2017).

9. Y. Zhang, L. Zhou, J. Gong, H. D. Ng, and H. Teng, "Effects of activation energy on the instability of oblique detonation surfaces with a one-step chemistry model," Phys. Fluids 30, 106110 (2018).

10. Y. G. Phylippov, V. R. Dushin, V. F. Nikitin, V. A. Nerchenko, N. V. Korolkova, and V. M. Guendugov, "Fluid mechanics of pulse detonation thrusters," Acta Astronaut. 76, 115 (2012).

11. J. Sun, J. Zhou, S. Liu, Z. Lin, and J. Cai, "Effects of injection nozzle exit width on rotating detonation engine," Acta Astronaut. 140, 388 (2017).

12. D. R. Cho, S. H. Won, J. R. Shin, and J. Y. Choi, "Numerical study of three-dimensional detonation wave dynamics in a circular tube," Proc. Combust. Inst. 34, 1929 (2013).

13. C. P. Li, and K. Kailasanath, "Detonation initiation by annular-jet-induced imploding shocks," J. Propul. Power 21, 183 (2005).

14. X. Han, J. Zhou, and Z. Lin, "Experimental investigations of detonation initiation by hot jets in supersonic premixed flows," Chin. Phys. B 21, 124702 (2012).

15. F. Carnasciali, J. H. S. Lee, R. Knystautas, and F. Fineschi, "Turbulent jet initiation of detonation," Combust. Flame 84, 170 (1991).

16. S. P. Medvedev, S. V. Khomik, and H. Olivier, "Hydrogen Detonation and Fast Deflagration Triggered by a Turbulent Jet of Combustion Products," Shock Waves 14, 193 (2005).

17. J. L. Hoke, R. P. Bradley, J. R. Gallia, and F. R. Schauer, "The impact of detonation initiation techniques on thrust in a pulsed detonation engine," AIAA (2006-1023).

18. S. Liu, Z. Lin, W. Liu, W. Lin, and F. Zhuang, "Experimental realization of $\mathrm{H}_{2} /$ air continuous rotating detonation in a cylindrical combustor," Combust. Sci. Technol. 184, 1302 (2012). 
19. J. Carpio, I. Iglesias, M. Vera, A. L. Sánchez, and A. Liñán, "Critical radius for hot-jet ignition of hydrogen-air mixtures," Int. J. Hydrogen Energy 38, 3105 (2013).

20. L. Wei, Z. Jin, S. Liu, Z. Lin, and F. Zhuang, "Experimental study on propagation mode of $\mathrm{H}_{2} / \mathrm{Air}$ continuously rotating detonation wave," Int. J. Hydrogen Energy 40, 1980 (2015).

21. X. Cai, R. Deiterding, J. Liang, M. Sun, and Y. Mahmoudi, "Diffusion and mixing effects in hot jet initiation and propagation of hydrogen detonations," Journal of Fluid Mechanics 863, 324 (2018).

22. W. Chen, J. Liang, X. Cai, and Z. Lin, "Detonation simulations in expanding channel with supersonic combustible mixture," Int. J. Hydrogen Energy 42, 6384 (2017).

23. X. Cai, R. Deiterding, J. Liang, and Y. Mahmoudi, "Adaptive simulations of viscous detonations initiated by a hot jet using a high-order hybrid WENO-CD scheme," Proc. Combust. Inst. 36, 2725 (2017).

24. X. Cai, J. Liang, R. Deiterding, Y. Che, and Z. Lin, "Adaptive mesh refinement based simulations of three-dimensional detonation combustion in supersonic combustible mixtures with a detailed reaction model," Int. J. Hydrogen Energy 41, 3222 (2016).

25. Y. Mahmoudi, and K. Mazaheri, "High resolution numerical simulation of triple point collision and origin of unburned gas pockets in turbulent detonations," Acta Astronaut. 115, 40 (2015).

26. A. Chinnayya, A. Hadjadj, and D. Ngomo, "Computational study of detonation wave propagation in narrow channels," Phys. Fluids 25, 036101 (2013).

27. E. Ioannou, S. Schoch, N. Nikiforakis, and L. Michael, "Detonation propagation in annular arcs of condensed phase explosives," Phys. Fluids 29, 116102 (2017).

28. B. V. Voitsekhovskii, V. V. Mitrofanov, and M. Y. Topchiyan, "The structure of a detonation front in gases," Technical Report FTD-MT-64-527(AD-633-821), 1966.

29. N. Tsuboi, K. Eto, and A. K. Hayashi, "Detailed structure of spinning detonation in a circular tube," 
Combust. Flame 149, 144 (2007).

30. C. Campbell, and D. W. Woodhead, "The ignition of gases by an explosion-wave. Part I. Carbon monoxide and hydrogen mixtures," J. Chem. Soc. 3010 (1926).

31. Z. W. Huang, M. H. Lefebvre, and P. J. V. Tiggelen, "Experiments on spinning detonations with detailed analysis of the shock structure," Shock Waves 10, 119 (2000).

32. V. N. Gamezo, T. Ogawa, and E. S. Oran, "Numerical simulations of flame propagation and DDT in obstructed channels filled with hydrogen-air mixture," Proc. Combust. Inst. 31, 2463 (2007).

33. V. N. Gamezo, A. Y. Poludnenko, E. S. Oran, and F. A. Williams, "Transverse waves resulting from pulsating instability of two-dimensional flames," Combust. Flame 161, 950 (2014).

34. D. N. Williams, L. Bauwens, and E. S. Oran, "Detailed structure and propagation of threedimensional detonations," Proc. Combust. Inst. 26, 2991 (1996).

35. N. Tsuboi, S. Katoh, and A. K. Hayashi, "Three-dimensional numerical simulation for hydrogen/air detonation: Rectangular and diagonal structures," Proc. Combust. Inst. 29, 2783 (2002).

36. C. Wang, C.-W. Shu, W. Han, and J. Ning, "High resolution WENO simulation of 3D detonation waves," Combust. Flame 160, 447 (2013).

37. C. Wang, P. Li, Z. Gao, and W. S. Don, "Three-dimensional detonation simulations with the mapped WENO-Z finite difference scheme," Comput. Fluids 139, 105 (2016).

38. R. Deiterding, and G. Bader, "High-resolution simulation of detonations with detailed chemistry," in Analysis and Numerics for Conservation Laws (Springer Berlin Heidelberg, 2005).

39. R. Deiterding, "Parallel adaptive simulation of multi-dimensional detonation structures," Ph.D. thesis, Brandenburg University of Technology, 2003.

40. R. Deiterding, "A parallel adaptive method for simulating shock-induced combustion with detailed chemical kinetics in complex domains," Comput. Struct. 11, 769 (2009). 
41. R. Deiterding, "High-resolution numerical simulation and analysis of mach reflection structures in detonation waves in low-pressure $\mathrm{H}_{2}-\mathrm{O}_{2}$-Ar Mixtures: A Summary of Results Obtained with the Adaptive Mesh Refinement Framework AMROC," J. Combust. (2011).

42. J. Liang, X. Cai, Z. Lin, and R. Deiterding, "Effects of a hot jet on detonation initiation and propagation in supersonic combustible mixtures," Acta Astronaut. 105, 265 (2014).

43. N. Tsuboi, and A. Koichi Hayashi, "Numerical study on spinning detonations," Proc. Combust. Inst. 31, 2389 (2007).

44. N. Tsuboi, A. K. Hayashi, and M. Koshi, "Energy release effect of mixture on single spinning detonation structure," Proc. Combust. Inst. 32, 2405 (2009).

45. Y. Sugiyama, and A. Matsuo, "Numerical investigation on propagation mechanism of spinning detonation in a circular tube," Proc. Combust. Inst. 32, 2331 (2009).

46. F. Virot, B. Khasainov, D. Desbordes, and H. Presles, "Spinning Detonation : Experiments and Simulations," in 21st International Colloquium on the Dynamics of Explosions and Reactive Systems (Poitiers, 2007).

47. E. S. Oran, and V. N. Gamezo, "Origins of the deflagration-to-detonation transition in gas-phase combustion," Combust. Flame 148, 4 (2007).

48. B. Varatharajan, and F. A. Williams, "Chemical-kinetic descriptions of high-temperature ignition and detonation of acetylene-oxygen-diluent systems," Combust. Flame 124, 624 (2001).

49. J. E. Shepherd, "Detonation in gases," Proc. Combust. Inst. 32, 83 (2009).

50. Z. Liang, and L. Bauwens, "Detonation structure with pressure-dependent chain-branching kinetics," Proc. Combust. Inst. 30, 1879 (2005).

51. M. Reynaud, F. Virot, and A. Chinnayya, "A computational study of the interaction of gaseous detonations with a compressible layer," Phys. Fluids 29, 056101 (2017). 
52. M. I. Radulescu, and B. M. Maxwell, "The mechanism of detonation attenuation by a porous medium and its subsequent re-initiation," Journal of Fluid Mechanics 667, 96 (2011).

53. G. B. Goodwin, R. W. Houim, and E. S. Oran, "Shock transition to detonation in channels with obstacles," Proc. Combust. Inst. 36, 2717 (2017).

54. J. L. Ziegler, R. Deiterding, J. E. Shepherd, and D. I. Pullin, "An adaptive high-order hybrid scheme for compressive, viscous flows with detailed chemistry," J. Comput. Phys. 230, 7598 (2011).

55. S. P. M. Bane, J. L. Ziegler, and J. E. Shepherd, "Development of One-Step Chemistry Models for Flame and Ignition Simulation," Technical Report GALCITFM:2010.002, 2010.

56. D. I. Kabanova, and A. R. Kasimovb, "A minimal hyperbolic system for unstable shock waves," Commun. Nonlinear Sci. Numer. Sim. 70, 282 (2019).

57. R. Deiterding, "Numerical simulation of transient detonation structures in $\mathrm{H}_{2}-\mathrm{O}_{2}$ mixtures in smooth pipe bends," in Proc. of the 21st International Colloquium on the Dynamics of Explosions and Reactive Systems ( Poitiers, 2007.7).

58. B. V. Leer, "On the relation between the upwind-differencing schemes of Godunov, Enguist-Osher and Roe," SIAM Jounarl on Scientific \& Statistical Computing 5, 1 (1985).

59. R. P. Fedkiw, T. Aslam, B. Merriman, and S. Osher, "A non-oscillatory Eulerian approach to interfaces in multimaterial flows (the ghost fluid method)," J. Comput. Phys. 152, 457 (1999).

60. S. P. Mauch, "Efficient Algorithms for Solving Static Hamilton-Jacobi Equations," California Institute of Technology, 2003.

61. X. Cai, J. Liang, R. Deiterding, and Z. Lin, "Adaptive simulations of cavity-based detonation in supersonic hydrogen-oxygen mixture," Int. J. Hydrogen Energy 41, 6917 (2016).

62. D. Goodwin, "Cantera: object-oriented software for reacting flows," Technical Report, 2005, available at http://www.cantera.org. 
63. F. Virot, B. Khasainov, D. Desbordes, and H. N. Presles, "Numerical simulation of the influence of tube diameter on detonation regime and structure in mixtures with two-step energy release and double cellular structure," Comb. Expl. Shock Waves 45, 435 (2009). 


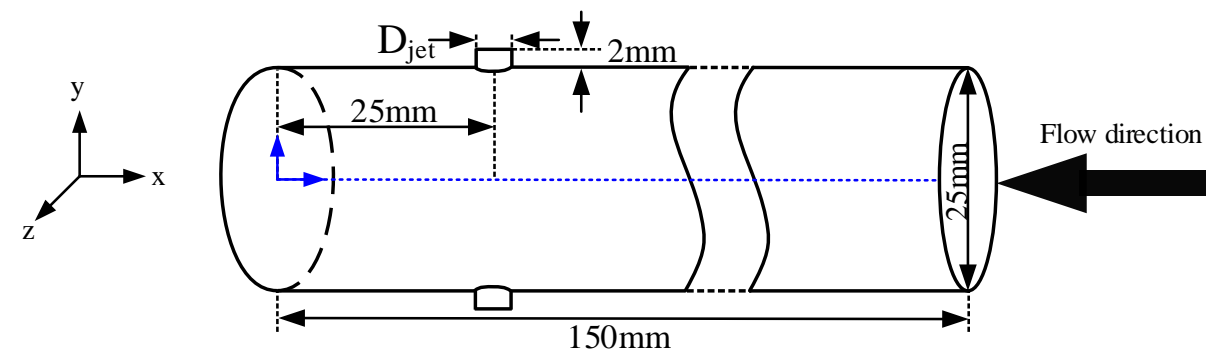

Fig. 1. Schematic of the computational domain. 

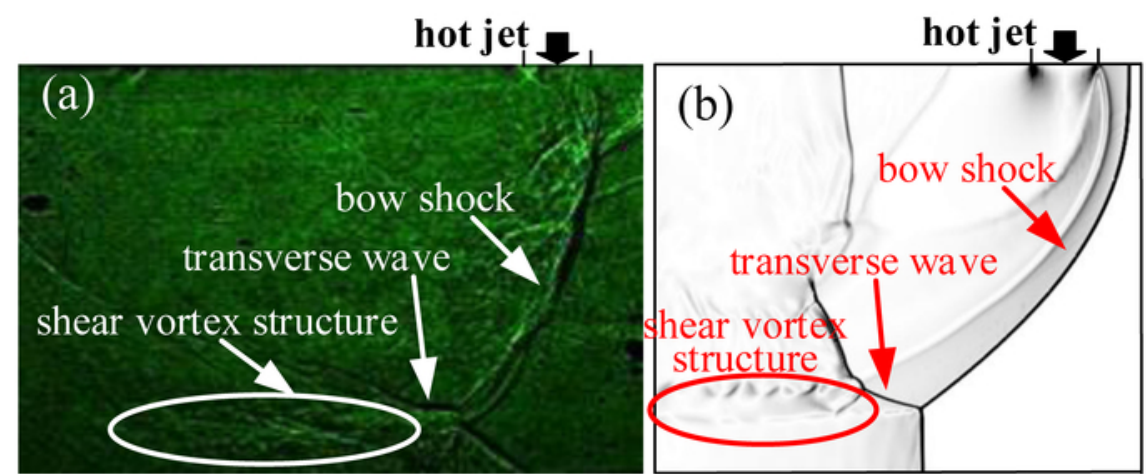

Fig. 2. Comparison between experimental result and numerical simulation. (a) experimental shadowgraph, (b) numerical schlieren of vertical center section calculated from xoy plane. 

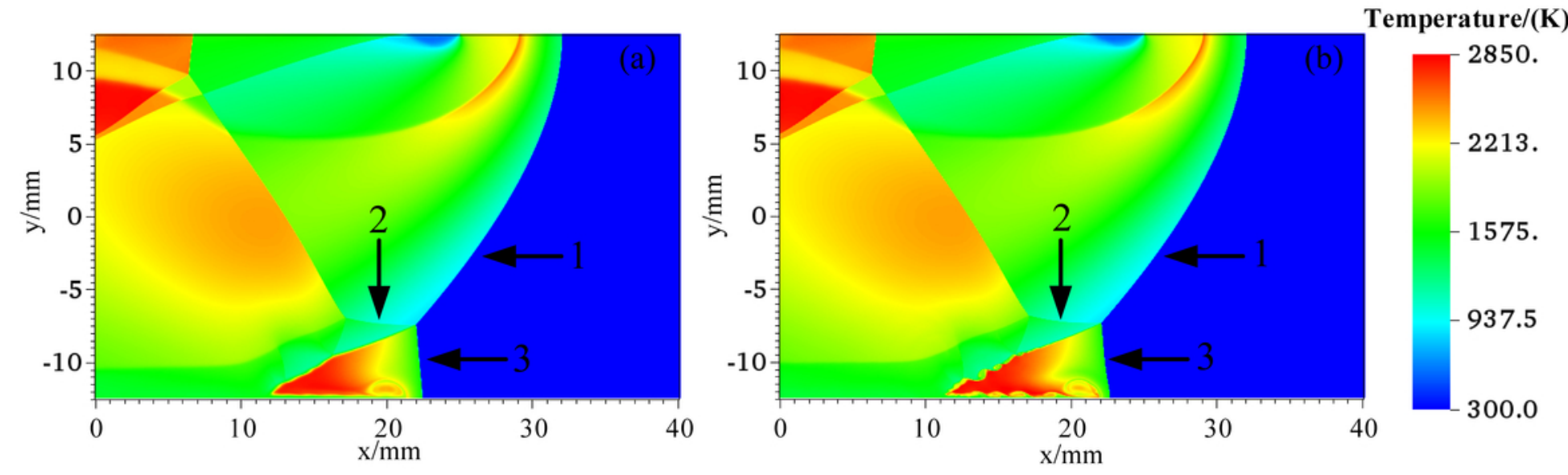

Fig. 3. Comparison of the initiation feature between (a) $57 \mathrm{Pts} / \mathrm{L}_{1 / 2}$ and (b) $114 \mathrm{Pts} / \mathrm{L}_{1 / 2}, 1$-bow shock, 2transverse wave, 3-Mach stem induced detonation. 

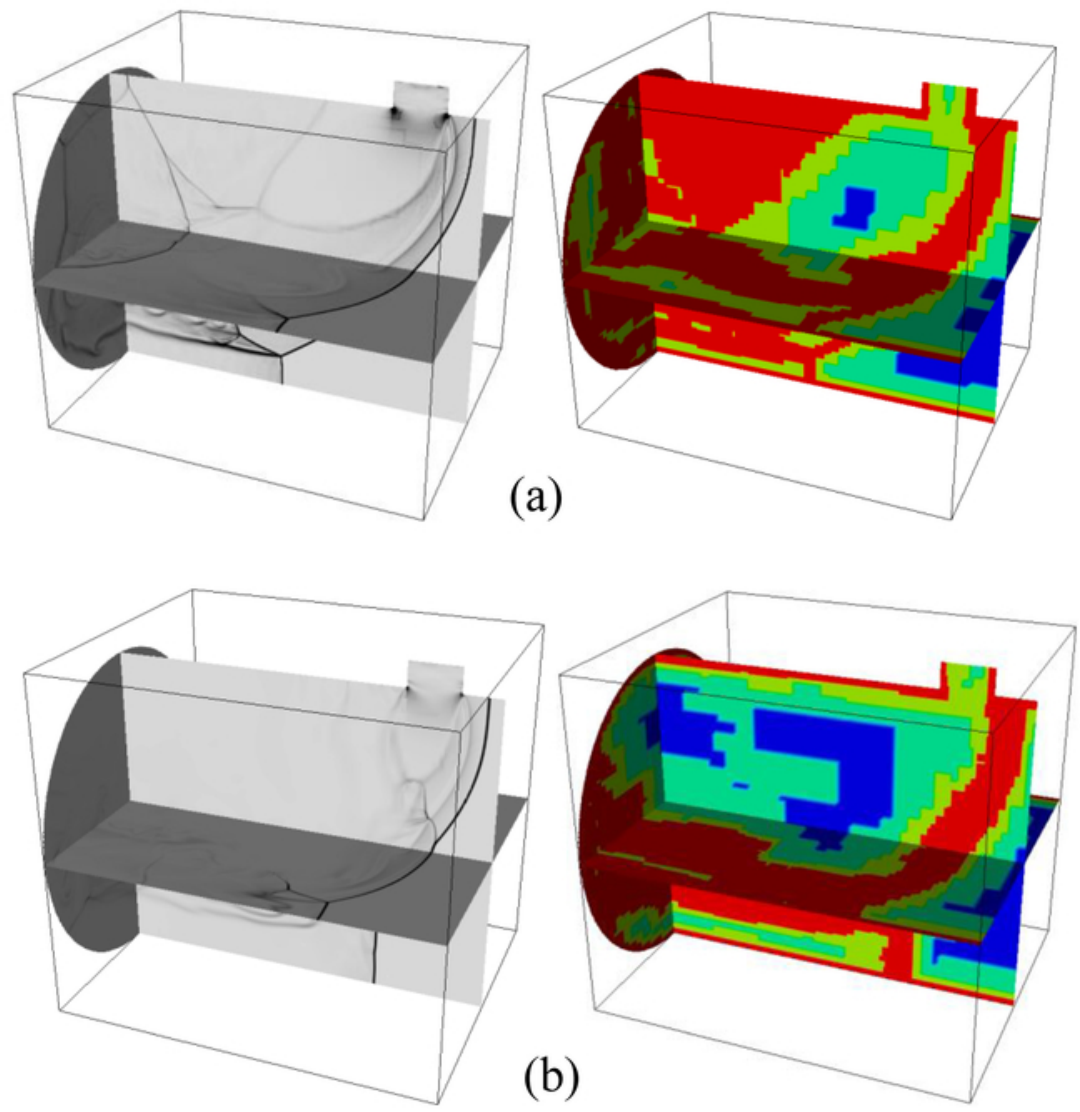

Fig. 4. Density schlieren images (left) and corresponding adaptive level distributions (right). (a) $t=105 \mu \mathrm{s}$, (b) $t=$ $180 \mu \mathrm{s}$. 


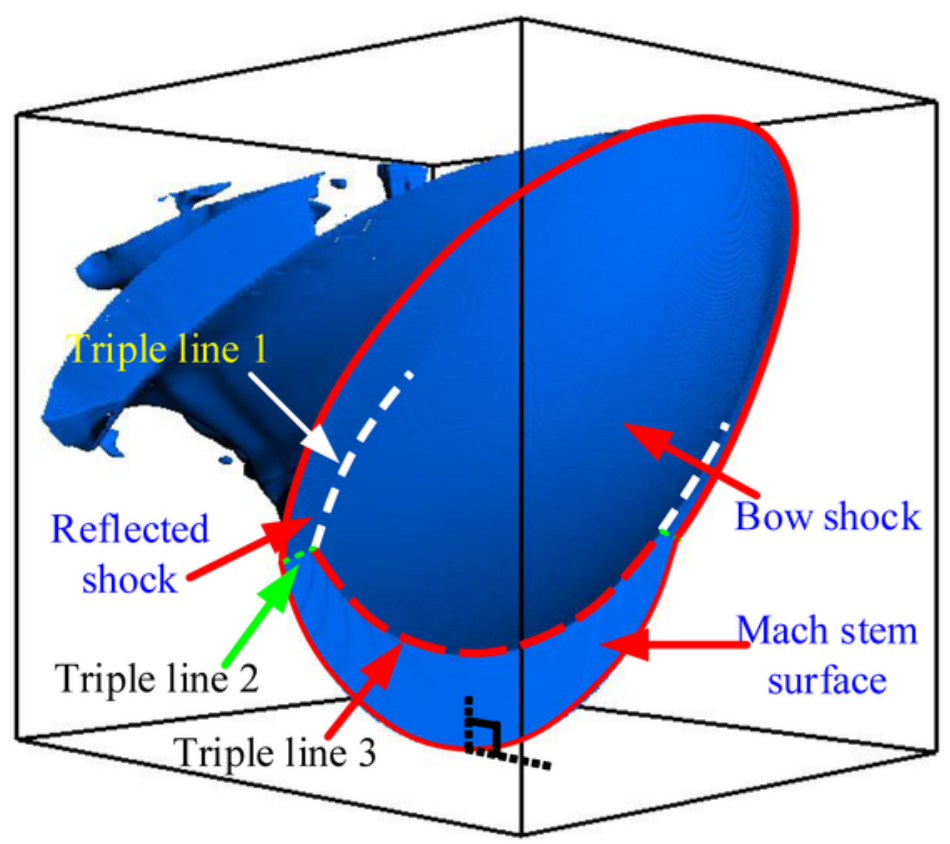

Fig. 5. Reflection structure of the jet-induced bow shock in the circular tube, $t=133 \mu$ s. 

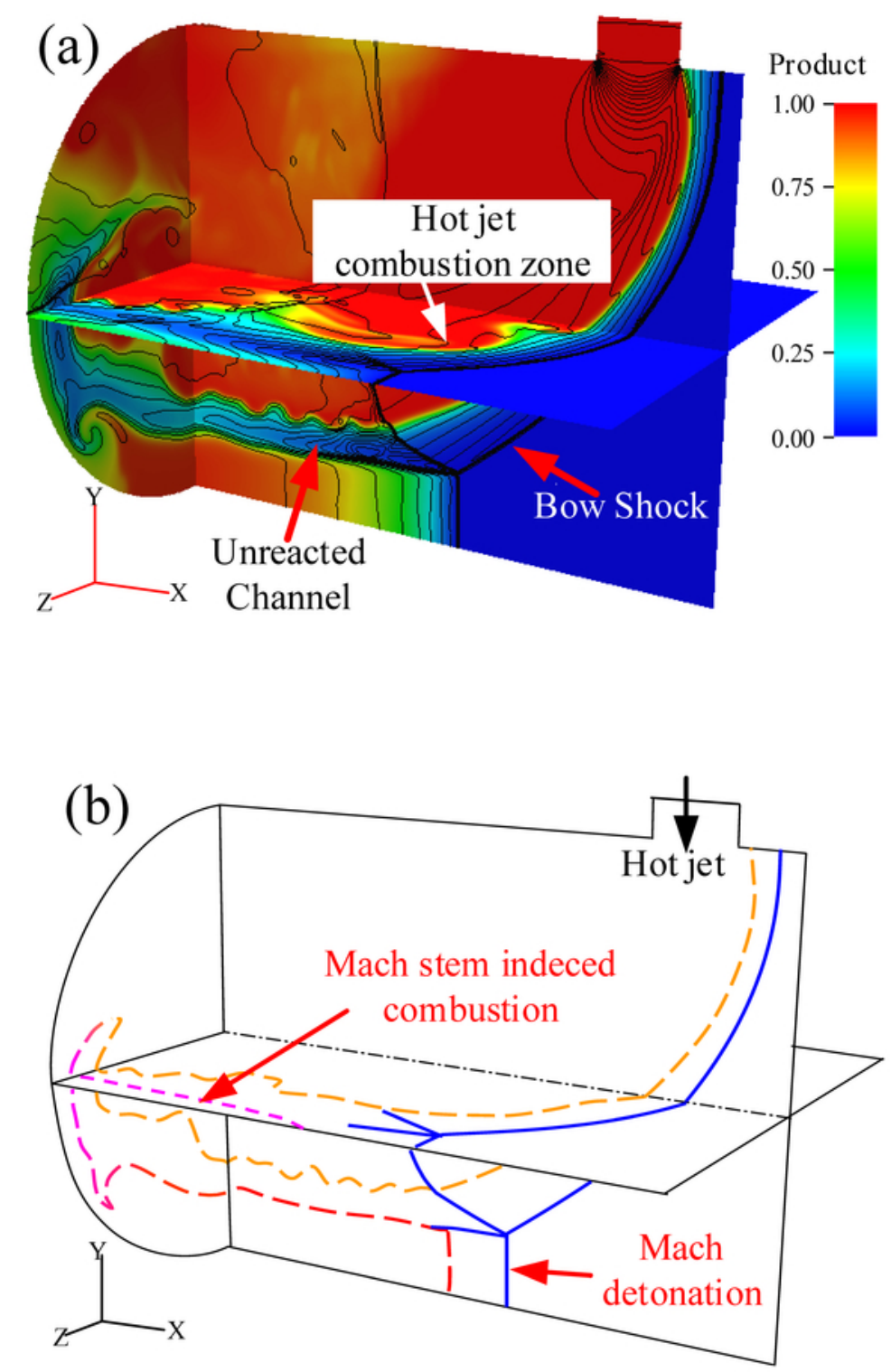

Fig. 6. Instantaneous structure of hot jet initiation at $t=133 \mu \mathrm{s}$ : (a) three slices on the contour of the product and density isolines and (b) schematic of the shock front and combustion front structure. 


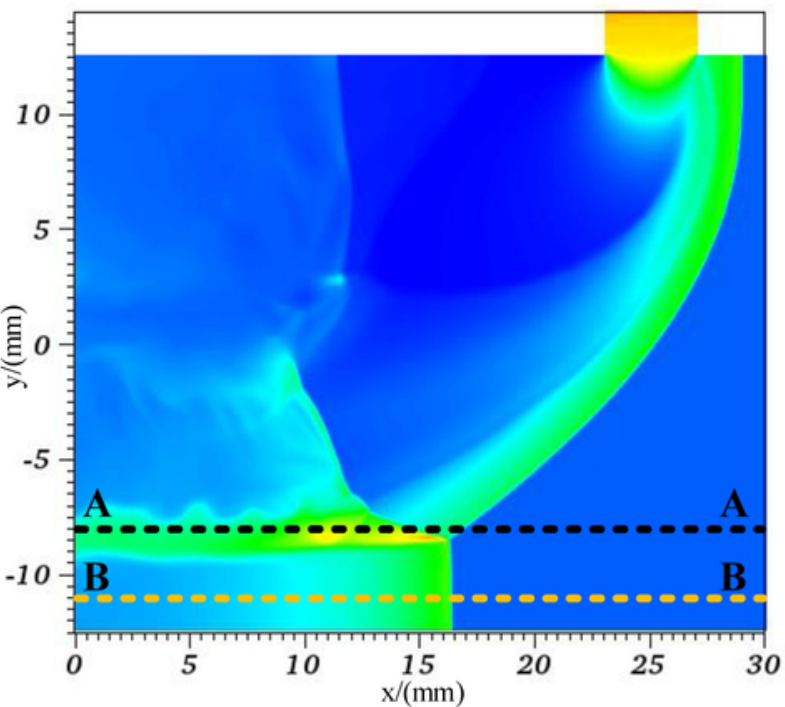

(a)
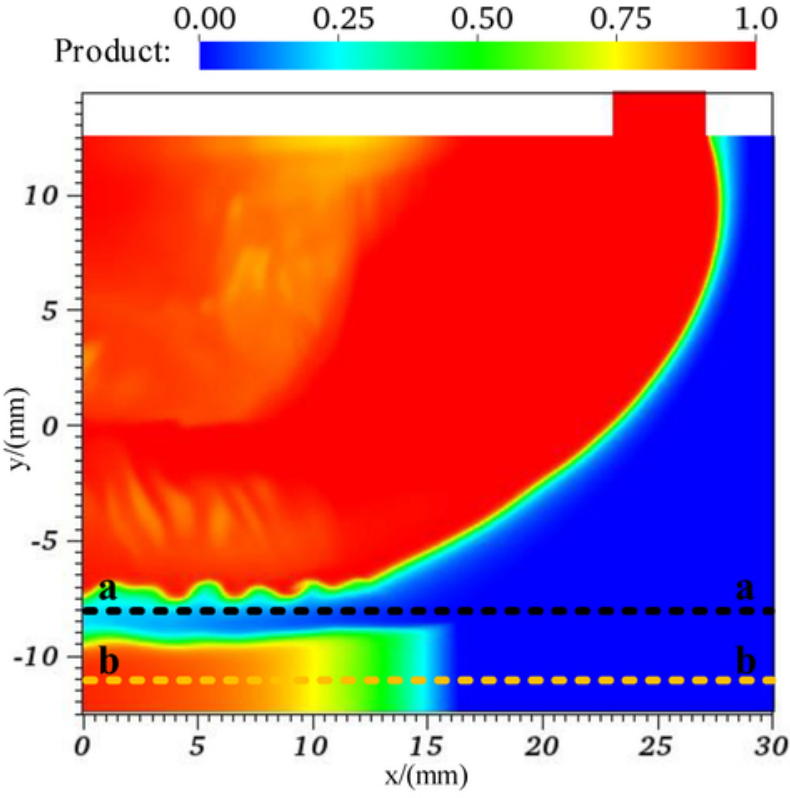

(c)

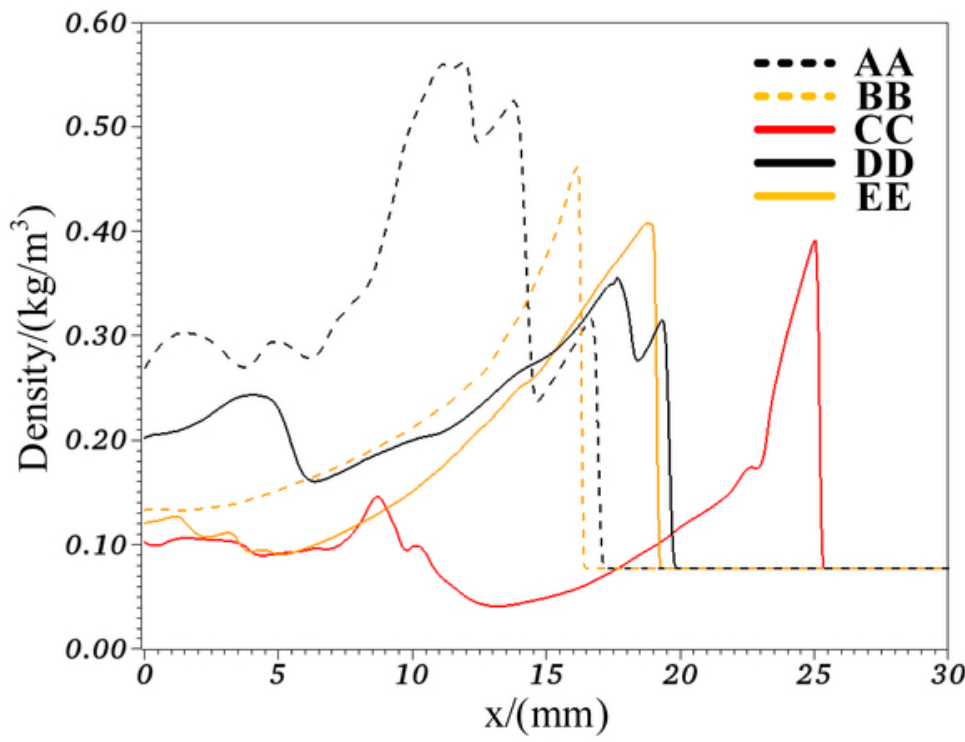

(e)

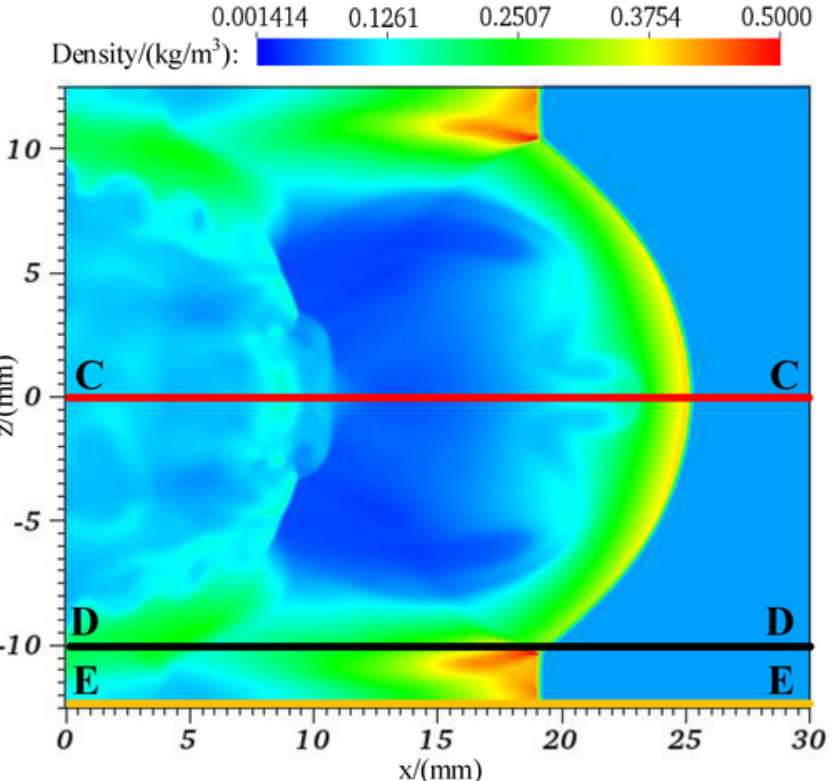

(b)

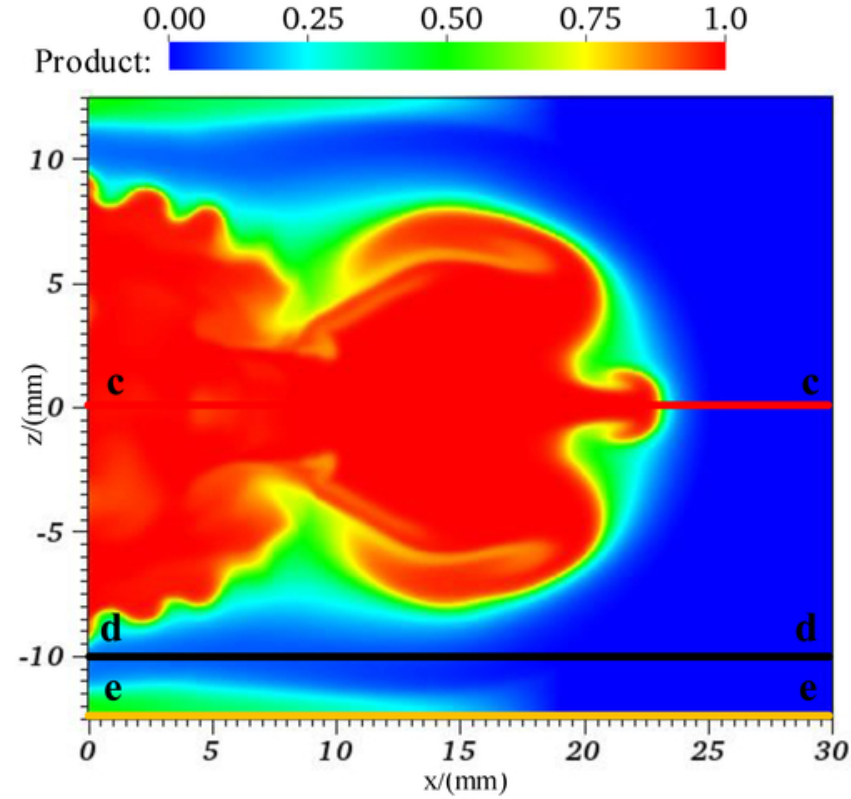

(d)

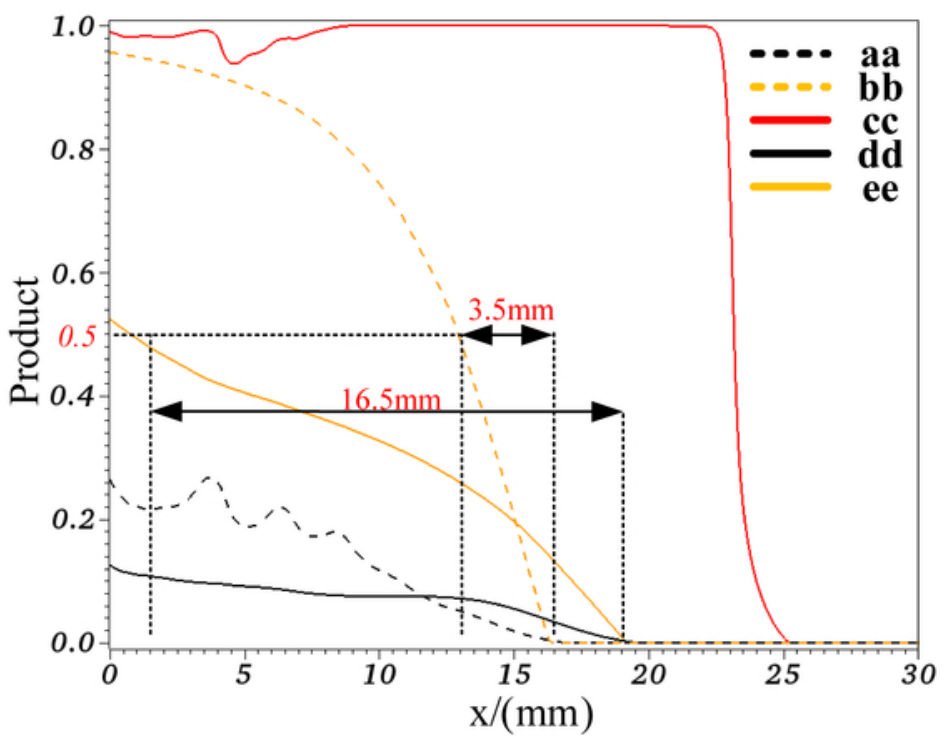

(f)

Fig. 7. Instantaneous density and product distributions of the flow fields at $t=133 \mu \mathrm{s}$, (a) the contour of density at vertical center section (xoy plane), (b) the contour of density at horizontal center section (xoz plane), (c) the contour of product at vertical center section (xoy plane), (d) the contour of product at horizontal center section (xoz plane), (e) instantaneous density curves at five positions shown in (a) and (b), (f) instantaneous product curves at five positions shown in (c) and (d). 


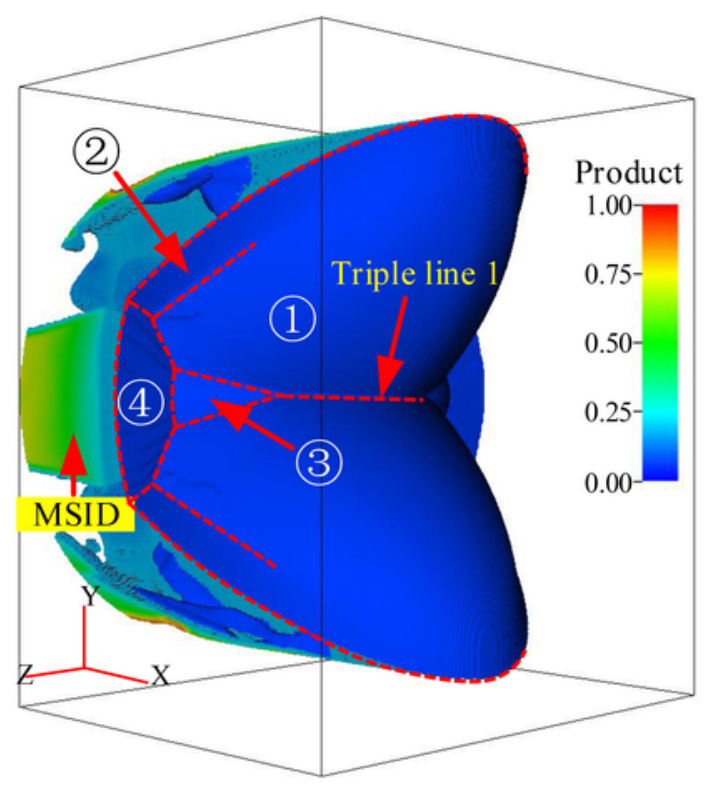

Fig. 8. Shock front structure of the impinging double hot jet-induced bow shock reflection at $t=130 \mu$ s. MSID: Mach stem-induced detonation, $\circ 1$ :bow shock, $\circ 2$ : Mach reflection on the circular tube, $\circ 3$ : Mach reflection by bow shocks interaction, $\circ 4$ :Mach reflection on the sidewall. 


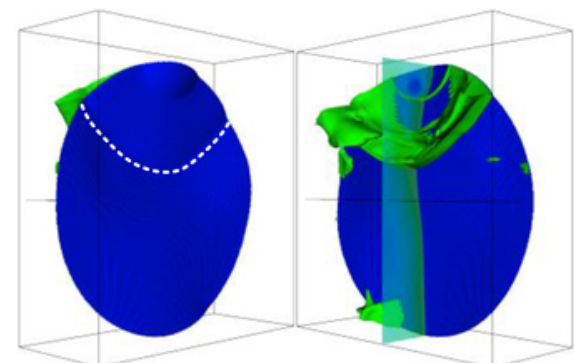

(a)

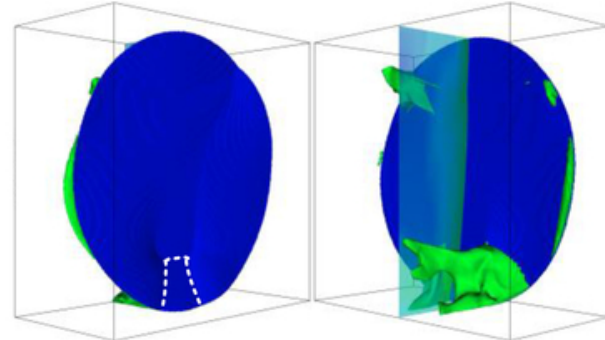

(c)

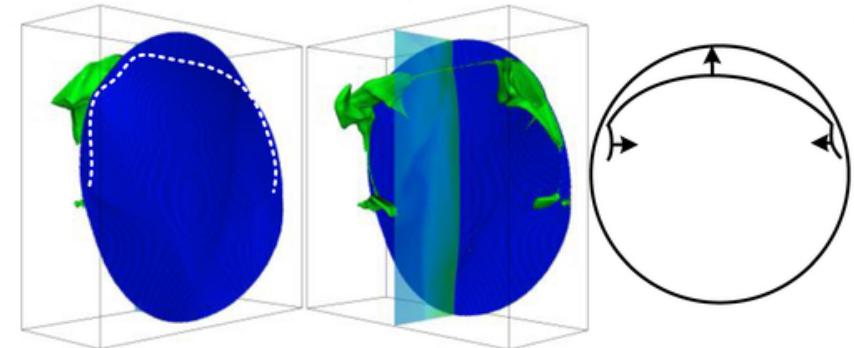

(e)
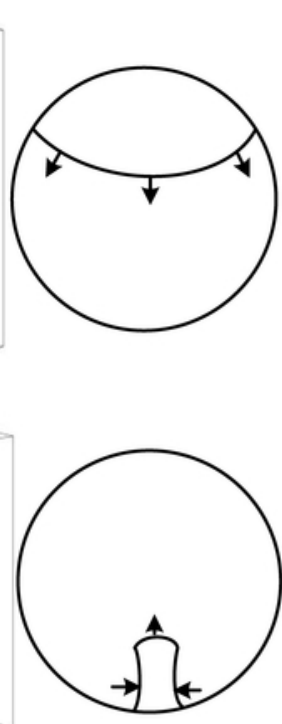

$+2$

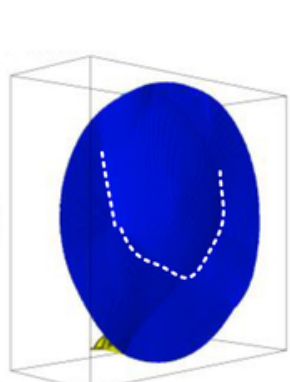

(1)

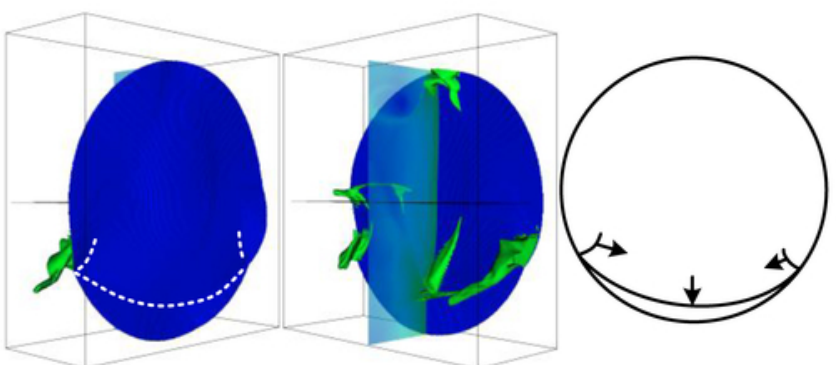

(b)

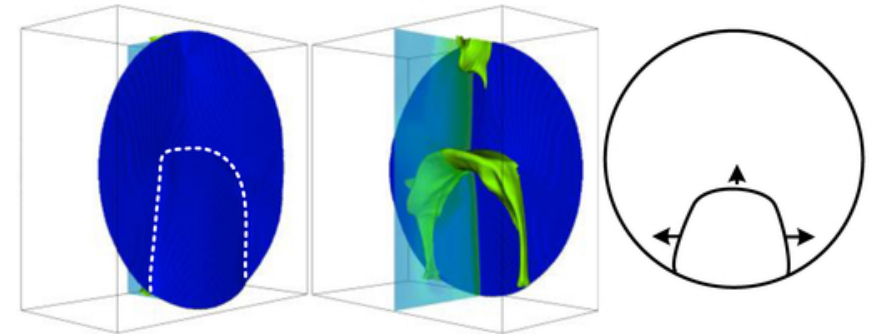

(d)

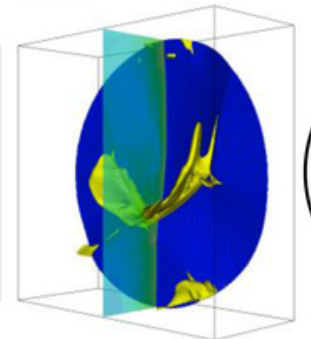

(f)

Fig. 9. Detonation propagation in the two-headed mode in a circular tube, (a) $t=220 \mu \mathrm{s}$, (b) $t=235 \mu \mathrm{s}$, (c) $t=245 \mu \mathrm{s}$, (d) $t=255 \mu \mathrm{s}$, (e) $t=265 \mu \mathrm{s}$, (f) $t=285 \mu \mathrm{s}$. The blue isosurface is the $0.09 \mathrm{~kg} / \mathrm{m}^{3}$ density isosurface, the green or lime yellow isosurface is the $245 \mathrm{kPa}$ pressure isosurface. 

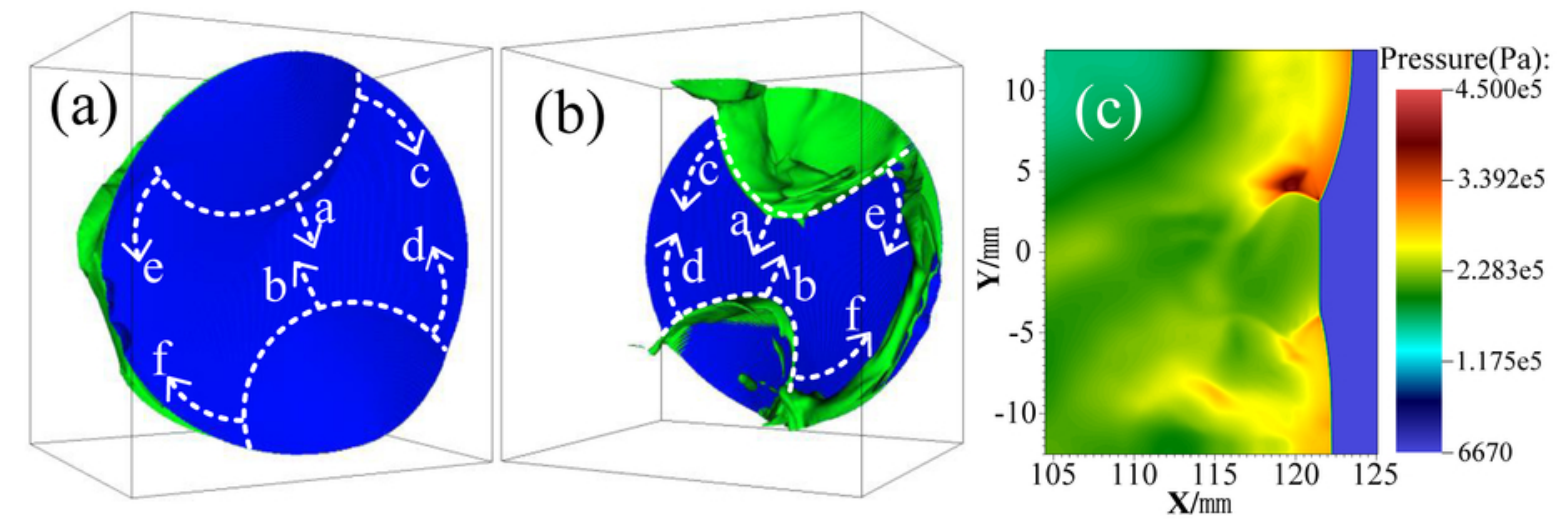

Fig. 10. Four-headed mode detonation wave front structures obtained with a higher inflow Mach number at $t=680$ $\mu \mathrm{s}$ : (a) front structure, (b) rear-side structure, and (c) cross-sectional structure. 


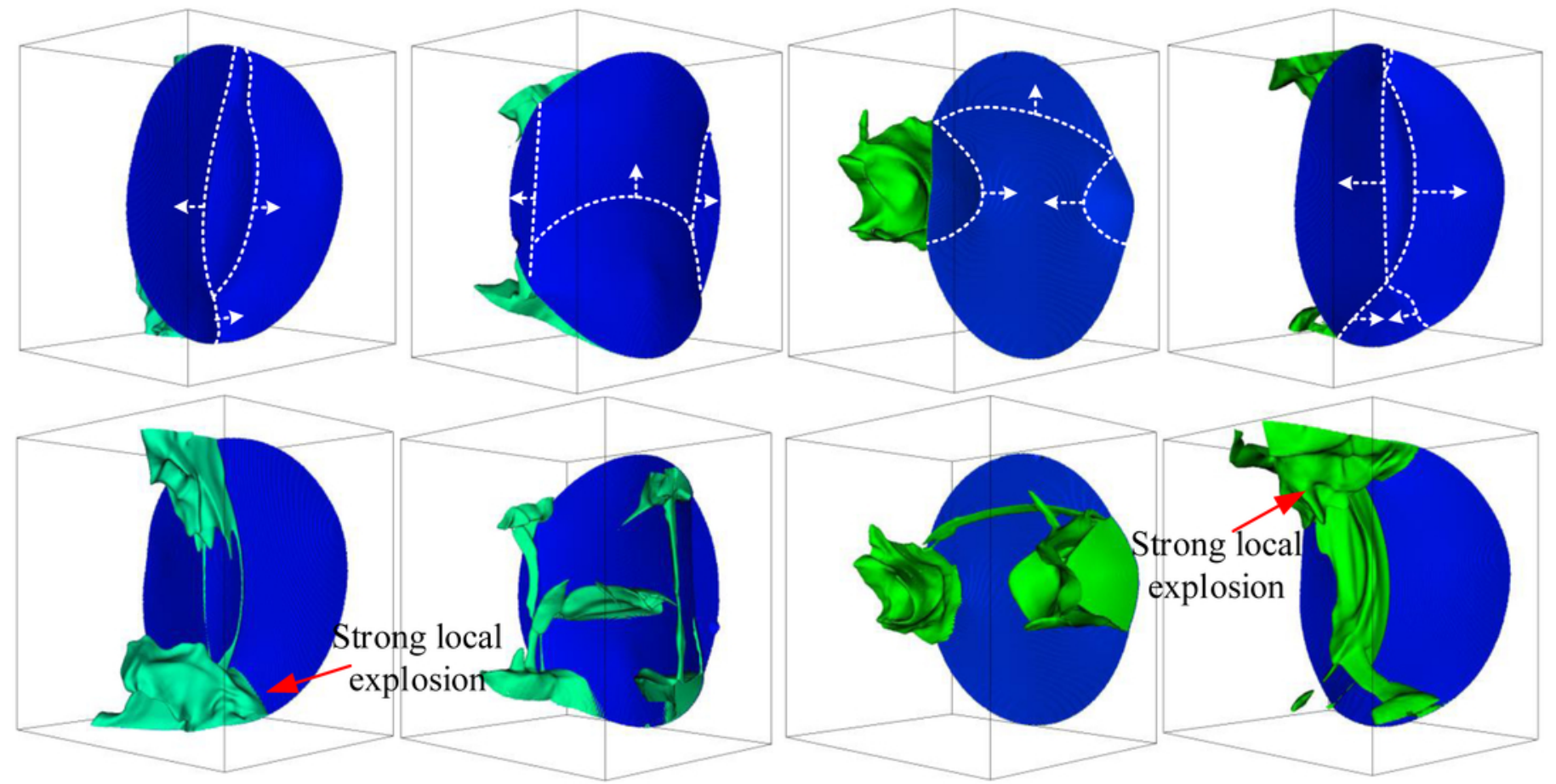

Fig. 11. Four-headed mode detonation wave front structures and cross-sectional structures obtained by impinging double hot jets. (a) $t=410 \mu \mathrm{s}$, (b) $t=420 \mu \mathrm{s}$, (c) $t=430 \mu \mathrm{s}$, (d) $t=440 \mu \mathrm{s}$. 